\title{
Pathogenic mechanisms and direction of empirical studies: a retrospective on membranoproliferative glomerulonephritis
}

\author{
Alexander Pazionis (Meds 2015) and Denise Darmawikarta (Meds 2016) \\ Faculty reviewer: Dr. Faisal Rehman, MD, FRCPC, M.Ed (Department of Medicine, Division of Nephrology)
}

$\mathrm{M}$ embranoproliferative glomerulonephritis (MPGN) is an immune-mediated disease process, underlying $10 \%-20 \%$ of nephrotic syndrome cases in children and young adults. ${ }^{1}$ The Type I subtype (Classical MPGN) is the most common Hepatitis C Virus $(\mathrm{HCV})$-associated nephropathy. ${ }^{2}$ Still, though all MPGN subtypes (I,II,III) can slowly progress to end-stage renal disease (ERDS), ${ }^{3}$ the condition is uncommon enough to account for just $2.8 \%$ and $3.3 \%$ of all cases in pediatric dialysis patients and pediatric renal transplant recipients, respectively. ${ }^{4}$ This may explain, in part, why studies on the efficacies of various immunosuppressive agents in this condition are, on the whole, relatively limited.

Nevertheless, the general findings (with exceptions) appear to indicate these agents are either ineffective or minimally effective in MPGN (depending on the subtype). In the current model of evidence-based medicine (EBM), it is understandable that much of clinical practice and research be guided by empirical findings. Nonetheless, when examining the theoretical pathogenesis of MPGN along with what is known of the mechanisms of action of these agents, it retrospectively becomes clear that some of these agents were unlikely to be effective in the first place. This is not to say that theoretical understanding should be trusted without empirical verification. However, in the same way that a clinician should consider what is known about an individual patient and order tests accordingly, it behooves the investigator to consider what is known about a condition before conducting an empirical study.

In this review, we discuss the underlying pathogenenic mechanisms of MPGN and review currently available treatment. We focus on idiopathic MPGN, as most cases of MPGN in adults have secondary causes (e.g. cancer, hepatitis C) and treatment would accordingly target those underlying conditions.

\section{PATHOGENIC MECHANISMS}

The complement cascade consists of a series of proteolytic steps catalyzed by the products of previous steps in the series. ${ }^{8}$ Figure 1 is a diagrammatic representation of the complement cascade, which can be activated via more than one pathway; the two most relevant to the discussion being the so-called 'classical' and 'alternative' pathways.

The classical pathway is part of the arsenal of the adaptive immune system's humoral arm..$^{8}$ The alternative pathway, on the other hand, is a part of non-specific or 'innate' immunity, and does not require antibodies, like the classical pathway does. ${ }^{8}$ However, after the cleavage of $\mathrm{C} 5$ to $\mathrm{C} 5 \mathrm{a}$ and $\mathrm{C} 5 \mathrm{~b}$, classical and alternative pathways converge to a common molecular sequence. ${ }^{8}$ It is at this stage, the so-called 'terminal' pathway that over-activation seems most highly associated with MPGN III. ${ }^{3,9,10}$ With this in mind, we may classify the subtypes of MPGN as follows:
Type I - Evidence of activation of the classical and alternative complement pathways, exhibiting on immunohistochemistry glomerular deposits of immunoglobulin (indicating immune complex deposition as a contributor to the disease process), as well as components unique to the classical complement cascade $(\mathrm{C} 1 \mathrm{q}, \mathrm{C} 4)$ in addition to those common to both the classical and alternative pathways (C3).

Type II - Also known as 'Dense Deposit Disease,' with conspicuous absence of components unique to the classical compliment cascade (C1q, C4) vis-à-vis absence of immunoglobulin deposition, while still maintaining evidence of $\mathrm{C} 3$ deposition in the kidney(s). ${ }^{1}$ The implication is a 'pure' alternative pathway component and a theoretical absence of an adaptive immunity contribution.

Type III - Less easily classified and arguably the rarest of the three commonly acknowledged subtypes. It is often considered a variation of Type I. It displays evidence of possible immune complex deposition as a mediator of glomerular damage via the observation of immunoglobulins on immunohistochemistry, but, as mentioned previously, is also highly associated with over activation of the terminal pathway of the complement cascade.

While the pathogenesis of MPGN is now becoming clearer, it is interesting to realize that currently employed therapeutic agents do not necessarily target components of the disease mechanisms.

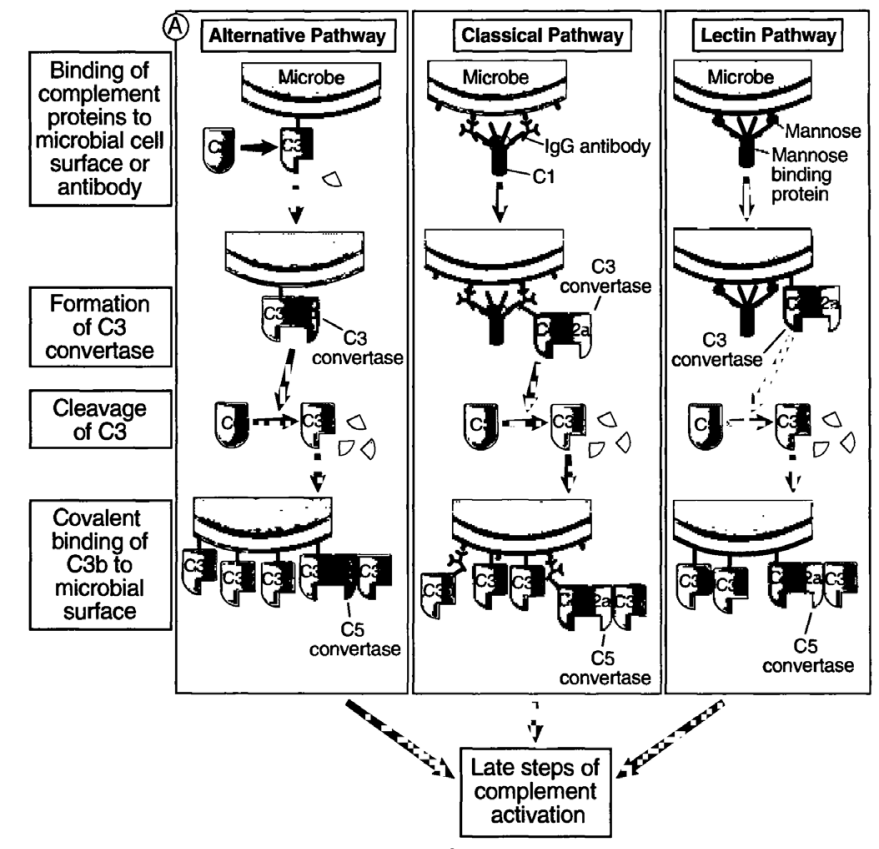

Figure 1. The complement cascade. ${ }^{8}$ 


\section{CLINICAL PROCEDURES CORTICOSTEROIDS}

An important study to consider in detail is a report of the International Study of Kidney Disease in Children. ${ }^{11}$ This study showed a statistically significant decrease in treatment failure for children with MPGN I and III, meeting their inclusion criteria when treated with prednisone rather than placebo $(\mathrm{p}=0.028)$. However, results were not statistically significant for children with MPGN II included in the study, though it was underpowered in this regard. This was corroborated by Donadio and Offord, ${ }^{12}$ whose study also failed to show benefit in adult MPGN II patients comparing prednisone to placebo. Finally, though not quite reaching statistical significance $(p=0.07)$, there was a trend toward stable, improved renal function considering all participants with MPGN I, II and III at 130 months of follow-up. ${ }^{11}$ A weakness of this study is that it did not differentiate between MPGN I and MPGN III under the assumption that MPGN III was a subcategory of MPGN I. Potential differences between these two groups were evaluated in a retrospective study by Braun et. al ${ }^{13}$ that showed statistically significant differences between a number of characteristics between MPGN I and MPGN III patients treated with prednisone at 3 years of follow up. Patients with MPGN I, compared with those having MPGN III, were found to have more stable GFR's, lower rates of persistent hypocomplimentemia (C3), and lower rates of persistent hematuria and proteinuria, to name a few.

Thus, with what evidence there is, a possible theoretical simplification is as follows with respect to the effectiveness of steroids in MPGN:

MPGN I - Effective

\section{MPGN III - Less Effective \\ MPGN II - Not Effective}

The effects of steroids are so categorically broad that any explanation on a mechanistic basis of these categories would amount to speculation. More simply put, it is difficult to identify differences in similar disease entities based on responses to a drug with broad effects. Other agents with greater specificity allow us to better distinguish such differences on the basis of theoretical knowledge. This is illustrated next, by the admittedly limited number of studies performed on calcineurin inhibitors.

\section{CALCINEURIN INHIBITORS}

Calcineurin mediates the activation of nuclear factor of activated t-cells (NFAT), a transcription factor encoding a number of genes including IL-2. ${ }^{8}$ IL-2 is necessary for T-cell proliferation. ${ }^{14}$ Other genes, such as CD40L and others required for T-cells to be able to activate B-cells are also produced. Blocking calcineurin, therefore, is expected to have a number of effects on the adaptive immune system including decreased antibody production and decreased T-cell inflammatory mediator release.

The possibility of using calcineurin inhibitors is best illustrated in two studies. The first, by Bagheri et. al. studied 18 patients with idiopathic MPGN (IMPGN) ${ }^{15}$. They showed that partial or complete remission of proteinuria was achieved in $94 \%(\mathrm{p}<0.01)$ of patients, and that at an average follow-up of 108 weeks, just one of those showed remission once treatment was discontinued ${ }^{15}$. Another study, by Singh et. al. similarly showed statistically significant reductions in proteinuria in 8 patients with MPGN treated with cyclosporine ${ }^{16}$. However, both of these studies failed to distinguish between different subtypes of MPGN. Bagheri et al. made no mention at all of different subtypes, and though Singh et al. disclosed that 5 of the MPGN patients were Type I and 3 were Type II, the data for all these patients were ultimately combined. The pathogenesis of MPGN I is directly linked to the classical pathway of compliment activation, which constitutes part of adaptive immunity and therefore is, in theory, potentially susceptible to inhibition by cal- cineurin inhibitors. This cannot be stated with certainty based on the studies discussed as they did not dissociate the effects of calcineurin inhibitors on type I vs. type II. MPGN II, on the other hand, is linked to innate immunity, and though there have been incidental case reports of MPGN II patients responding to cyclosporine ${ }^{14}$, long-term followup studies of small numbers of patients show that calcineurin inhibitors have no impact on renal survival in MPGN II patients ${ }^{17}$. Long-term renal survival in MPGN I patients treated with cyclosporine should be further investigated.

\section{CONCLUSION/FUTURE DIRECTION}

While some evidence exists that suggests a role for other treatments, such as acetylsalicylic acid, dipyridamole, cyclophosphamide and Coumadin (warfarin), in MPGN, it is unfortunately of poor quality ${ }^{18}$. Randomized controlled trials should be conducted to study these various treatment options for MPGN. Given the general ineffectiveness of current MPGN treatment, it is imperative that future investigations on potential therapeutic agents be conducted on the basis of known pathogenic mechanisms.

A promising treatment option for MPGN that has been elucidated by case reports 5-7 is Eculizumab. Eculizumab is a monoclonal antibody for $\mathrm{C} 5$, a member of the complement cascade that forms part of the MAC. Given the pathogenesis of MPGN, the use of Eculizumab makes intuitive sense and thus it is unfortunate that little is yet known about their effectiveness in the treatment of MPGN. Insofar as C5 is involved in both the classical and alternative pathways of complement activation, all three subtypes of MPGN (which, somewhat simplified, may be characterized by differential ratios of classical:alternative pathway activation), could theoretically be treated with this medication. Future studies should therefore investigate the potential for Eculizumab in treating MPGN, insofar as potentially, all three subtypes of MPGN could be treated with this agent.

\section{REFERENCES}

1. Kumar V, Abbas AK, Fausto N, Aster JC, editors. Pathologic Basis of Disease. 8th ed. Philadelphia: Elsevier; 2010.

2. Fabrizi F. Hepatitis C virus, cryoglobulinemia, and kidney: Novel evidence. Scientifica. 2012;2012

3. Alchi B, Jayne D. Membranoproliferative glomerulonephritis. Pediatr Nephrol. 2010 Aug;25(8):1409-18.

4. Warady BA, Hebert D, Sullivan EK, Alexander SR, Tejani A. Renal transplantation, chronic dialysis, and chronic renal insufficiency in children and adolescents. the 1995 annual report of the north american pediatric renal transplant cooperative study. Pediatr Nephrol. 1997 Feb;11(1):49-64.

5. Daina E, Noris M, Remuzzi G. Eculizumab in a patient with dense-deposit disease. N Engl J Med. 2012 Mar 22;366(12):1161-3.

6. Vivarelli M, Pasini A, Emma F. Eculizumab for the treatment of dense-deposit disease. N Engl J Med. 2012 Mar 22;366(12):1163-5.

7. Radhakrishnan S, Lunn A, Kirschfink M, Thorner P, Hebert D, Langlois V, Pluthero F, Licht C. Eculizumab and refractory membranoproliferative glomerulonephritis. N Engl J Med. 2012 Mar 22;366(12):1165-6.

8. Abbas AK, Lichtman AH, editors. Basic Immunology: Functions and Disorders of the Immune System. 3rd ed. Philadelphia: Elsevier; 2011.

9. Clardy CW, Forristal J, Strife CF, West CD. Serum terminal complement component levels in hypocomplementemic glomerulonephritides. Clin Immunol Immunopathol. 1989 Mar;50(3):307-20.

10. Varade WS, Forristal J, West CD. Patterns of complement activation in idiopathic membranoproliferative glomerulonephritis, types I, II, and III. Am J Kidney Dis. 1990 Sep;16(3):196-20.

11. Tarshish P, Bernstein J, Tobin JN, Edelmann CM,Jr. Treatment of mesan- 
giocapillary glomerulonephritis with alternate-day prednisone--a report of the international study of kidney disease in children. Pediatr Nephrol. 1992 Mar;6(2):123-30.

12. Donadio JV,Jr, Offord KP. Reassessment of treatment results in membranoproliferative glomerulonephritis, with emphasis on life-table analysis. Am J Kidney Dis. 1989 Dec;14(6):445-51.

13. Braun MC, West CD, Strife CF. Differences between membranoproliferative glomerulonephritis types I and III in long-term response to an alternate-day prednisone regimen. Am J Kidney Dis. 1999 Dec;34(6):1022-3.

14. Ho S, Clipstone N, Timmermann L, Northrop J, Graef I, Fiorentino D, Nourse J, Crabtree GR. The mechanism of action of cyclosporin A and FK506. Clin Immunol Immunopathol. 1996 Sep;80(3 Pt 2):S40-5.

15. Bagheri N, Nemati E, Rahbar K, Nobakht A, Einollahi B, Taheri S. Cyclosporine in the treatment of membranoproliferative glomerulonephritis. Arch Iran Med. 2008 Jan;11(1):26-9.

\section{CLINICAL PROCEDURES}

16. Singh A, Tejani C, Tejani A. One-center experience with cyclosporine in refractory nephrotic syndrome in children. Pediatr Nephrol. 1999 Jan;13(1):2632.

17. Appel GB, Cook HT, Hageman G, Jennette JC, Kashgarian M, Kirschfink M, Lambris JD, Lanning L, Lutz HU, Meri S, Rose NR, Salant DJ, Sethi S, Smith RJ, Smoyer W, Tully HF, Tully SP, Walker P, Welsh M, Wurzner R, Zipfel PF. Membranoproliferative glomerulonephritis type II (dense deposit disease): An update. J Am Soc Nephrol. 2005 May;16(5):1392-403.

18. Longo DL, Kasper DL, Jameson JL, Fauci AS, Hauser SL, Loscalzo J, editors. Harrison's Principles of Internal Medicine. 18th ed. United States of America: McGraw-Hill Professional; 2012.

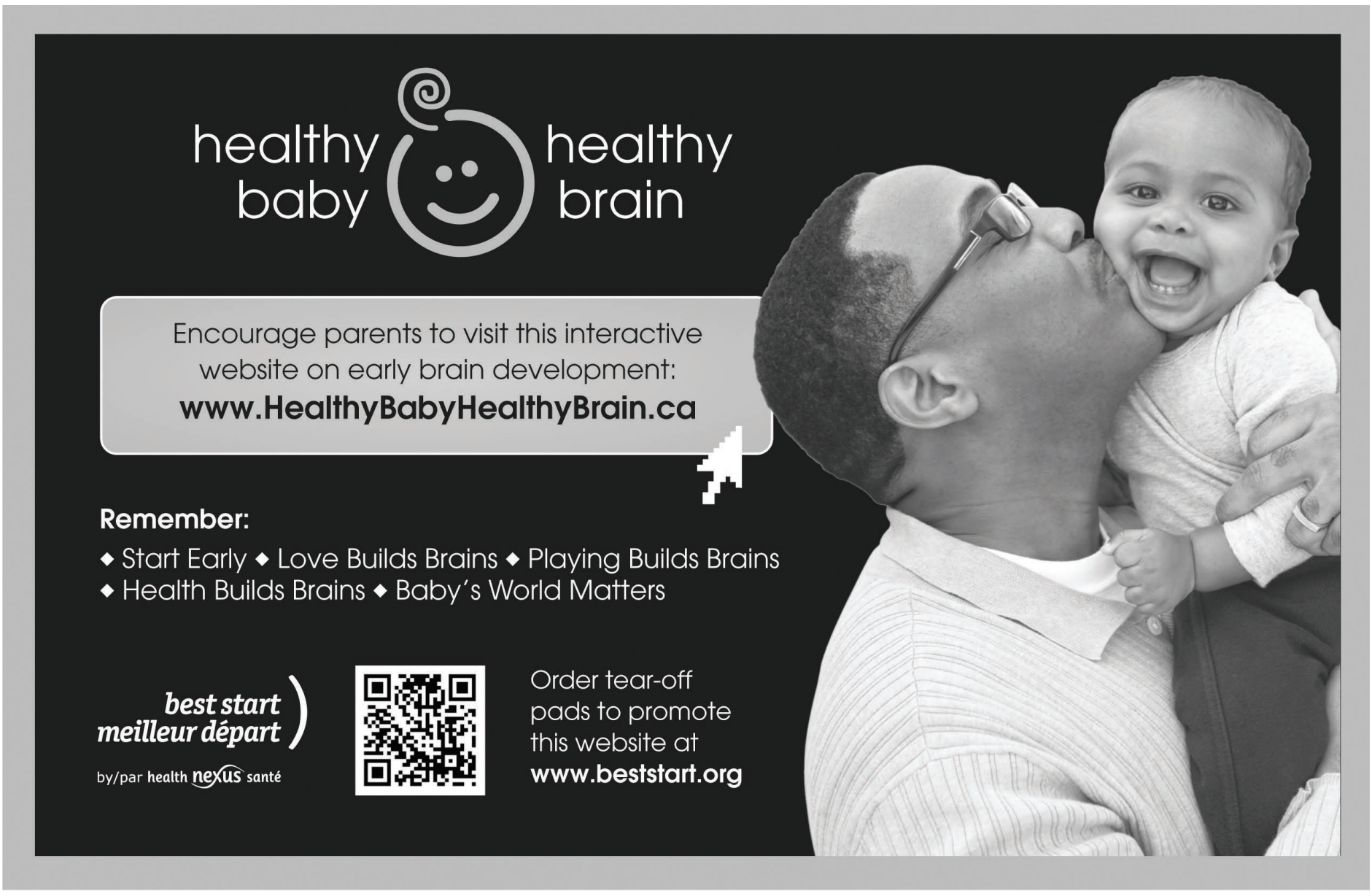

